\section{Tratamiento endoscópico en la pancreatitis crónica: seguimiento a largo plazo}

\author{
ALEX DÍAZ ${ }^{1}$, PAOLA YUNGE ${ }^{1}$, ÁLVARO URZÚA ${ }^{1}$, ZOLTÁN BERGER $^{1,2}$
}

\section{Endoscopic treatment in chronic pancreatitis: long-term results in 18 patients}

\begin{abstract}
Background: Intraductal stones, ductal abnormalities and pancreatic pseudocysts are part of chronic pancreatitis (CP). The goal of treatment is pain relief, resolution of local complications and relapse prevention. Endoscopic therapy (ET) can be considered in those who do not respond to medical treatment. Aim: To evaluate the indication, immediate and long-term results of ET in CP patients. Patients and Methods: Review of a database of patients with CP analyzing results of ET in 18 patients aged 16 to 60 years (13 males). Demographics, etiology, endoscopic technique, indication for treatment, pain relief, relapses and complications were recorded. Results: The etiology of CP was alcohol consumption in 5, idiopathic in 11, hereditary in one and autoimmune in one case. The follow-up period was 6 months to 14 years. Seven patients had diabetes mellitus type $3 c$ and eight had moderate to severe exocrine pancreatic insufficiency. Pancreatic papillotomy was performed in all patients, with removal of some stones, without attempting a complete clearance of the pancreatic duct. In addition, a 7-10 French stent was placed in the main pancreatic duct in 15 patients with varying permanence (months to years). The stent was changed guided by recurrence of clinical symptoms. During the follow-up period, 10 patients remained asymptomatic and in three, pain or relapse were significantly reduced. Stenting failed in one patient for technical reasons. Two patients were operated. There were neither immediate nor late complications from ET. Conclusions: Long-lasting improvement of CP was observed in 13 of 18 patients treated with ET, without complications associated with the procedure.
\end{abstract}

(Rev Med Chile 2015; 143: 1121-1128)

Key words: Laparoscopy; Pancreatitis, chronic; Pain.

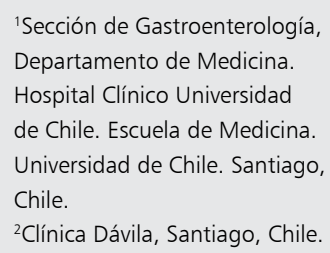

Correspondencia a:

Dr. Zoltán Berger

Hospital Clínico Universidad

de Chile, Sección de

Gastroenterología, Departamento

de Medicina.

Teléfono: 29788350

berger.zoltan@gmail.com

\section{L}

a pancreatitis crónica (PC) es un proceso inflamatorio crónico del páncreas, caracterizado por la presencia de infiltrado inflamatorio crónico, activación de células estrelladas pancreáticas con producción de fibrosis parenquimatosa y pérdida progresiva de células acinares y, posteriormente, islotes de Langerhans ${ }^{1}$. La evolución es característicamente progresiva, lo que lleva a un daño irreversible que conduce a la fibrosis del tejido pancreático, desdiferenciación de las células acinares, formación de complejos tubulares $^{2,3}$. La prevalencia de esta patología es variable según la literatura ${ }^{4}$, en Chile no existen cifras epidemiológicas fidedignas, pero la PC es considerada como una enfermedad rara, sino excepcional. La cronicidad de la PC y sus frecuentes reagudizaciones genera un impacto significativo en la calidad de vida del paciente.

Actualmente, se considera la pancreatitis crónica como un proceso multifactorial, en el que la presencia de uno o más factores de riesgo condiciona el desarrollo de la enfermedad. Entre 
estos factores se incluyen los tóxicos (alcohol y tabaco), metabólicos (hipercalcemia), genéticos (mutaciones en genes PRSS1, SPINK1, CFTR y CTCR, entre otros), inmunológicos-inflamatorios y obstructivos ${ }^{2,5-8}$. El alcohol sigue siendo la causa más común de PC en el mundo occidental. Sarles et al informaron que entre 60 y $70 \%$ de los pacientes tienen aproximadamente 20 o más años de historia de abuso de alcohol' .

El cuadro clínico de la PC varía desde la evolución asintomática hasta el dolor invalidante, puede presentar complicaciones que interfieren con la calidad de vida e incluso pueden hasta amenazar la vida misma ${ }^{10,11}$. El tratamiento de los síntomas de la enfermedad es preferentemente los menos invasivo posible, conservador. Esta conducta está avalada por la evidencia que muestra una evolución a largo plazo hacia la disminución de síntomas y complicaciones ${ }^{12}$, llegando a una etapa "burned-out", con destrucción avanzada del parénquima, cuando la insuficiencia exo y endocrina domina el cuadro clínico, sin mayores complicaciones $^{12}$. Sin embargo, antes de llegar a este nivel de destrucción del tejido pancreático, el dolor intratable o las recurrencias, en una proporción variable de pacientes, pueden requerir un tratamiento invasivo, tradicionalmente la cirugía pancreática descompresiva o resectiva, con buenos resultados clínicos ${ }^{13-15}$. Por otro lado, desde hace 3 a 4 décadas, aparece la alternativa menos invasiva, el tratamiento endoscópico (TE), que ha ganado un importante terreno en el manejo ${ }^{6,16-21}$. Como la PC es rara en Chile, sólo existen algunas publicaciones de pacientes que recibieron TE con éxito técnico y varios de estos enfermos tuvieron buena respuesta a corto y mediano plazo $^{22-23}$.

El objetivo de este trabajo fue describir y analizar la evolución de nuestros pacientes portadores de PC tratados con TE en dos centros asistenciales de Santiago.

\section{Pacientes y Métodos}

Estudio retrospectivo-descriptivo, efectuado con la base de datos de pacientes con PC del Hospital Clínico de la Universidad de Chile y de la Clínica Dávila. Analizamos 18 pacientes con PC sometidos a TE. Se registraron los datos demográficos, etiología, técnica endoscópica efectuada, indicación del tratamiento, nivel de mejoría del dolor, presencia y número de recaídas, y las complicaciones asociadas al procedimiento endoscópico. El diagnóstico de la pancreatitis crónica se estableció según criterios conocidos. Todos los pacientes incluidos en este trabajo, tuvieron alteraciones morfológicas severas compatibles con el diagnóstico, demostradas por tomografía computada (TAC) o resonancia magnética (RM). Además, como parte del procedimiento terapéutico, colangio-pancreatografía retrógrada endoscópica (CPRE) fue realizada, confirmando las alteraciones ductales compatibles con el diagnóstico. La función exocrina fue estudiada por elastasa fecal. Glicemia en ayuno, o antecedente de diabetes mellitus fue registrado. El consumo del alcohol fue considerado como factor etiológico, si el paciente hubiera consumido más de 35 bebidas alcohólicas/semana durante años ${ }^{24}$. Factor hereditario está sólo planteado en un caso, donde el padre y nuestra paciente hija son portadores de PC. No se estudiaron mutaciones.

La indicación de tratamiento endoscópico fue determinada por evolución clínica: dolor intratable, que requirió uso frecuente de opiáceos u hospitalizaciones, sin mejoría significativa con dieta y terapia oral con enzimas pancreáticas. Otra indicación era la recurrencia frecuente, más de tres episodios de pancreatitis aguda demostrada al año, que no cambió con dieta adecuada o abstinencia completa.

El método endoscópico consistió en realizar una pancreatografía endoscópica, papilotomía pancreática y colocar stent (Figura 1) con la excepción de dos casos, donde el objetivo era sólo realizar esfinterotomía doble y en otro, quisto-gastrostomía endoscópica. El proyecto fue aceptado por Comité de Ética de Hospital Clínico Universidad de Chile y de la Clínica Dávila.

\section{Resultados}

Entre los años 1995 y 2014, registramos 120 casos de pancreatitis crónica. De ellos, 18 pacientes fueron tratados por métodos endoscópicos, 13 hombres y 5 mujeres, con un promedio de edad global de 39,6 años (intervalo de 16-60 años) (Tabla 1). El consumo de alcohol fue considerado como el factor etiológico en 5 pacientes, no pudimos identificar una causa conocida en 10 pacientes, calificando a ellos como PC idiopática. 


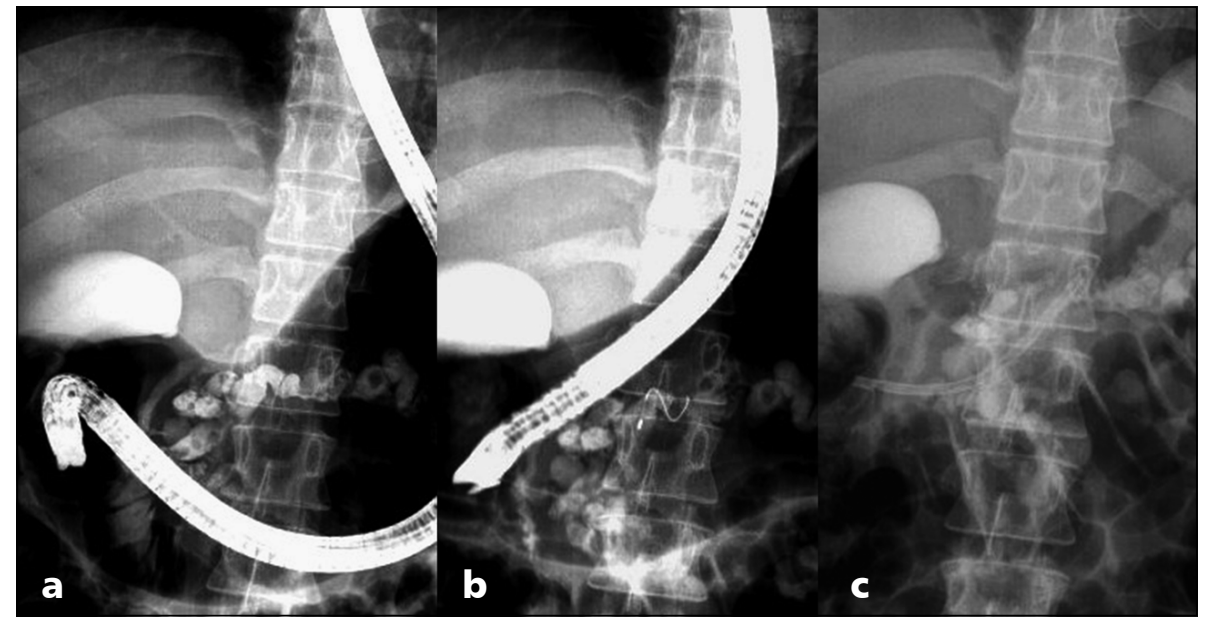

Figura 1. a) Conducto pancreático principal canulado y contrastado por la papila menor, dilatado, con múltiples defectos de llene, cálculos; b) Guía introducida en el conducto pancreático principal; c) Endoprótesis pancreática.

Tabla 1. Características de los pacientes y su respuesta al tratamiento endoscópico

\begin{tabular}{|c|c|c|c|c|c|c|c|}
\hline Género & Edad & Etiología & Recaídas & $\begin{array}{l}\text { Conducto } \\
\text { pancreático }\end{array}$ & TE & $\begin{array}{l}\text { Stent } \\
\text { años }\end{array}$ & Mejoría, años \\
\hline $\mathrm{H}$ & 30 & Alcohol & Sí & Dilatado, cálculos & $\mathrm{EC}+\mathrm{EP}$ & 1 & Completa, 8 años \\
\hline $\mathrm{H}$ & 35 & Alcohol & Sí & Dilatado, cálculos & $\mathrm{EC}+\mathrm{EP}$ & 4 & Completa, 8 años \\
\hline $\mathrm{H}$ & 18 & Idiopática & Sí & Dilatado, cálculos & $\mathrm{EC}+\mathrm{EP}$ & 5 & Completa, 7 años \\
\hline$H^{*}$ & 22 & Idiopática & Sí & Dilatado, cálculos & $\mathrm{EC}+\mathrm{EP}$ & $5+2$ & Completa, 11 años \\
\hline $\mathrm{H}$ & 19 & Idiopática & No & Dilatado, cálculos & $\mathrm{EC}+\mathrm{EP}$ & 1 & Parcial, sin control \\
\hline $\mathrm{H}^{* *}$ & 25 & Idiopática & Sí & Irregular, no dilatado & EP 2 veces & - & No, cirugía \\
\hline $\mathrm{H}$ & 35 & Idiopática & No & Dilatado, cálculos & $\mathrm{EC}+\mathrm{EP}$ & 1 & Completa, 2 años \\
\hline $\mathrm{H}$ & 60 & Idiopática & No & Estenosis dominante & EP & 0,5 & Completa 5 años \\
\hline $\mathrm{H}$ & 56 & Autoinmune & No & Estenosis dominante & EP & 0,5 & Completa, 4 años \\
\hline $\mathrm{H}^{*}$ & 16 & Idiopática & Sí & Cálculos, dilatación & EP & 0,5 & Parcial, 0,5 \\
\hline M & 58 & Idiopática & No & Estenosis dominante & EP & 1 & Completa, 7 años \\
\hline$M^{*}$ & 42 & Idiopática & Sí & $\begin{array}{l}\text { Dilatado, sin, luego } \\
\text { con cálculos }\end{array}$ & $\mathrm{EC}+\mathrm{EP}$ & 5 & Completa, 5 años \\
\hline$M^{*}$ & 53 & Idiopática & No & Dilatado, cálculos & $\mathrm{EC}+\mathrm{EP}$ & 1 & Parcial, 1 año \\
\hline$M^{* *}$ & 25 & Hereditaria & Sí & Dilatado, cálculos & $\mathrm{EC}+\mathrm{EP}$ & $4+1$ & $\begin{array}{l}\text { Completa, luego no, } \\
10 \text { años }\end{array}$ \\
\hline M & 53 & Idiopática & Sí & Dilatación, estenosis & EP & 0,5 & Completa, 2 años \\
\hline $\mathrm{H}$ & 56 & Alcohol & No & Cálculos, dilatación & Fracaso técnico, BC & - & 8 años, completa \\
\hline $\mathrm{H}$ & 22 & Alcohol & Sí & Diskinesia hipertónica & Papilotomía doble & & Completa \\
\hline $\mathrm{H}$ & 26 & Alcohol & Sí & Pseudoquiste & Quistogastrostomía & - & Pérdida de control \\
\hline
\end{tabular}

Todos los pacientes, con la excepción del último (pseudoquiste) fueron sometidos a CPRE, papilotomía pancreática. TE: tratamiento endoscópico; EC: extracción de cálculos; EP: endoprótesis (stent); BPC: bloqueo de plexo celíaco; *sigue con stent; **operado, ambos casos pancreato-duodenectomía. 
La PC fue hereditaria en una paciente y de origen autoinmune en 1. La diabetes mellitus tipo III c insulino-requirente se encontró asociada en 7 casos, insuficiencia exocrina moderada a severa fue demostrada en 8 casos. La indicación de TE fue por dolor en los 18 pacientes, asociándose con recaídas agudas frecuentes en 11 pacientes y con un pseudoquiste grande, sintomático en un paciente. En este último paciente se realizó quisto-gastrostomía endoscópica-posteriormente se perdió de control. En otro paciente la intervención endoscópica fue sólo papilotomía doble (biliar y pancreática), siendo demostrada previamente diskinesia hipertónica del esfínter biliar y dominantemente pancreático con manometría del esfínter de Oddi. Su evolución a largo plazo fue muy buena, sin embargo, no se puede considerar como efecto de TE, porque paralelamente dejó de consumir alcohol. En el resto de los pacientes, se realizó papilotomía pancreática, seguido con extracción de algunos cálculos, en 6 hombres y en 3 mujeres, sin pretender limpiar completamente el conducto pancreático. Se instaló stent 7 o 10F al conducto pancreático en 15 pacientes, con una permanencia variable ( 6 meses a 7 años). La instalación del stent fracasó en un paciente por razones técnicas, por lo que se efectuó bloqueo del plexo celiaco por radiólogo intervencionista, con buen resultado clínico. En otro paciente el stent no dio resultado clínico, ni en el corto plazo, y fue retirado en algunos días. En su caso, el conducto pancreático no fue dilatado. El paciente fue sometido a una pancreato-duodenectomía, con buena respuesta clínica.

Los cambios de stent se efectuaron según necesidad, considerando la recurrencia del dolor como indicador para elegir el momento del cambio. En cuanto al resultado clínico asociado a la TE, encontramos que 14 de los 15 pacientes respondieron con mejoría inmediata al TE. De ellos, 10 pacientes se mantuvieron asintomáticos durante años, y se redujo el dolor en 3 pacientes, ya permitiendo su manejo sin opiáceos y no se observaron más recaídas. Sólo un paciente no respondió nunca al TE: él no tenía conducto dilatado y finalmente fue operado, realizándose una pancreatoduodenectomía, con excelente resultado clínico.

Después de un período asintomático largo, en 11 casos por más de un año, se extrajo el stent. Se observó resolución de la estenosis previa en 2 pacientes, en el resto no encontramos cambio morfológico evaluable. El dolor recurrió en 3 pacientes. Dos de ellos respondieron nuevamente con gran mejoría a nueva intervención endoscópica con colocación de nuevo stent. Desgraciadamente, la paciente portadora de PC hereditaria, ya no respondió a nuevo intento endoscópico, ni a dos bloqueos de plexo celiaco y terminó en una pancreatoduodenectomía, con buena evolución posterior.

Actualmente, 4 de nuestros pacientes están con stent pancreático, 2 asintomáticos, 2 con gran mejoría, mínimas molestias.

El seguimiento fue entre 0,5-14 años. No observamos complicaciones inmediatas, ni tardías asociadas a la TE.

\section{Discusión}

La PC es una enfermedad que puede ser incapacitante, con complicaciones graves que afectan a la calidad de vida ${ }^{4,5,10,11,25}$. El objetivo del tratamiento de PC es el control de los síntomas. Presencia de cálculos intraductales, alteraciones demostradas de conducto pancreático sin síntomas clínicos, sin complicaciones no requieren intervenciones. El tratamiento de la insuficiencia exocrina y endocrina está bien establecido, el reemplazo de las enzimas y de insulina cuya producción es deficiente. Sin embargo, las reagudizaciones recurrentes y el dolor invalidante pueden requerir tratamiento invasivo ${ }^{15,17,21,25}$. La causa de las recaídas puede ser morfológica, detectable con métodos apropiados: alteraciones en la función esfinteriana, estrecheces del conducto, complicaciones locales, pseudoquistes ${ }^{16}$. El dolor es aun más complejo: no se comprende exactamente su causa. El alza en la presión intraductal, con elevación intraparenquimatosa secundaria, es una teoría antigua y no completamente rechazada ${ }^{8}$. Sin embargo, no hay relación clara entre la presión y el dolor, tampoco en la reducción de la presión por colocación de stent y la respuesta clínica ${ }^{7}$. Cambios histológicos de nervios intra y peripancreáticos fueron demostrados en PC en relación con el dolor ${ }^{27}$. Cirugía descompresiva o resectiva ha sido la terapia tradicional en estos casos difíciles, con respuesta clínica aceptable en diferentes series $^{15}$. Sin embargo, apareció el tratamiento menos invasivo, endoscópico. Los objetivos del tratamiento endoscópico son aliviar la obstrucción del flujo del conducto pancreático para disminuir 
la hipertensión ductal, drenar las colecciones de líquido, aliviar el dolor y prevenir las recaídas ${ }^{17,28,31}$. Las modalidades endoscópicas disponibles son: el tratamiento de las estenosis pancreáticas, de los cálculos ductales pancreáticos, de las estenosis del conducto biliar y de los pseudoquistes ${ }^{6,29,30}$. Las técnicas utilizadas son diversas y no difieren mayormente de las técnicas utilizadas cotidianamente en la endoscopia terapéutica biliar: la papilotomía pancreática, extracción instrumental de cálculos, colocación de endoprótesis, etc. El bloqueo de plexo celíaco para manejo del dolor puede efectuarse por endosonografía (EUS $)^{16}$ o por radiología intervencional ${ }^{32}$. La terapia endoscópica puede considerarse en los pacientes que no responden al tratamiento médico ${ }^{16}$, con alteraciones del conducto pancreático dilatado. La realización de estas intervenciones, técnicamente, no es más difícil que los procedimientos biliares. A diferencia de la colangiopancreatografía endoscópica retrógrada (CPRE) habitual, cuando se intenta evitar la canulación del conducto pancreático o, por lo menos, inyectar mínima cantidad de medio de contraste en el Wirsung, en estos casos el objetivo es la buena visualización y la adecuada manipulación en el conducto pancreático. La pancreatitis es una complicación temida de CPRE con indicaciones tradicionales, páncreas normal, pero excepcional en la TE de PC, cuando la destrucción del páncreas es ya avanzada y la manipulación en el conducto generalmente bien tolerada. De hecho, no hemos observado complicación alguna en nuestra serie, lo que concuerda con las experiencias publicadas.

La colocación de stent pancreático profiláctico es una recomendación después de una canulación difícil de la vía biliar y es una práctica cada vez más aceptada. En estos casos el stent es de 3-5 F, se mantiene en el conducto pancreático, por algunos días con el objetivo de asegurar el flujo libre de la secreción desde el conducto pancreático venciendo el edema transitorio de la papila. En cambio, en la terapia endoscópica de PC, el uso de endoprótesis obedece a similar lógica que en la vía biliar e incluye la intención de dilatar la estenosis. Por consiguiente, las prótesis son de 7-10 F, se mantienen por largo tiempo en el conducto y eventualmente se puede colocar múltiples stent-que no usamos en nuestros pacientes. Las intervenciones endoscópicas resultaron en una descompresión del conducto pancreático, con resultados clínicos excelentes: la mayoría de pacientes evolucionó bien con el tratamiento endoscópico. La excepción fue un paciente sin mayor dilatación de conducto pancreático. Aun en su caso, la ausencia de respuesta ayudó en tomar decisión quirúrgica y elegir resección en vez de intentar descompresión quirúrgica: resulta poco probable obtener una respuesta positiva, si la descompresión con método endoscópico fue ineficiente. Quedan las preguntas: ¿Cuál es el momento adecuado para el cambio del stent? ¿Cuándo se puede intentar retirar el stent y dejar al paciente sin la prótesis pancreática? En nuestra práctica, no cambiamos la prótesis en forma programada, nos guiamos por la sintomatología clínica. Esta conducta nos parece lógica y mucho más barata que los cambios regulares trimestrales sugeridos por algunos grupos ${ }^{6,33}$. El stent pancreático se tapa rápidamente, pero cumple al mantener permeable y dilatado el segmento estrecho del conducto, sin provocar lesiones histológicas severas. Tampoco existe método exacto para definir el retiro del stent: después de largo período asintomático intentamos dejar al paciente sin nueva prótesis, de hecho, sólo 4 pacientes se encuentran actualmente con prótesis pancreática, el resto ya evoluciona bien sin stent en el momento del análisis.

Existe evidencia que apoya el manejo endoscópico, con resultados variables. La TE es clínicamente eficaz para $50 \%$ de los pacientes con pancreatitis crónica sintomática. Cuando la TE no tiene éxito, la cirugía tiene resultados exitosos en $50 \%$ de los pacientes ${ }^{15}$, es decir, $75 \%$ de pacientes responden favorablemente a un tratamiento invasivo. Nuestros resultados clínicos han sido aun algo superiores: sólo un paciente fue operado por fracaso del TE, sin estenosis dominante, sin dilatación mayor del conducto principal. Estos hallazgos morfológicos y la ausencia de respuesta clínica a la colocación de stent permitieron elegir cirugía resectiva sin intentar descompresión. En otra de nuestras pacientes, el TE dio muy buenos resultados durante largos años, se logró mantenerla sin stent y clínicamente muy bien. Sin embargo, después de algunos años sin stent, nuevamente presentó dolor intratable, recaídas agudas dolorosas y finalmente terminó con una pancreato-duodenectomía, con buen resultado clínico.

En resumen: La indicación de tratamiento invasivo en PC es siempre clínica: el dolor, las recaídas o las complicaciones locales, que no 
responden al tratamiento médico. Frente a las molestias persistentes, recomendamos realizar colangioresonancia. Si el conducto pancreático principal es normal, se puede intentar realización de bloqueo del plexo celíaco. Si existe sospecha de tumor maligno, se considera resolución quirúrgica sin otros exámenes adicionales. Cuando el conducto pancreático está dilatado, con o sin cálculos, la TE puede ser la primera opción terapéutica. Si la respuesta es positiva, se mantiene el stent y se cambia según necesidad clínica, mientras en caso de fracaso clínico, la cirugía resectiva puede significar la mejor alternativa (Figura 2).

La TE es una herramienta útil, tiene bajo riesgo de complicaciones, con buena respuesta en PC con conducto principal dilatado, cálculo obstructivo o estenosis dominante. Nuestros resultados avalan que la TE bien indicada y en pacientes adecuadamente seleccionados puede postergar o reemplazar la cirugía.

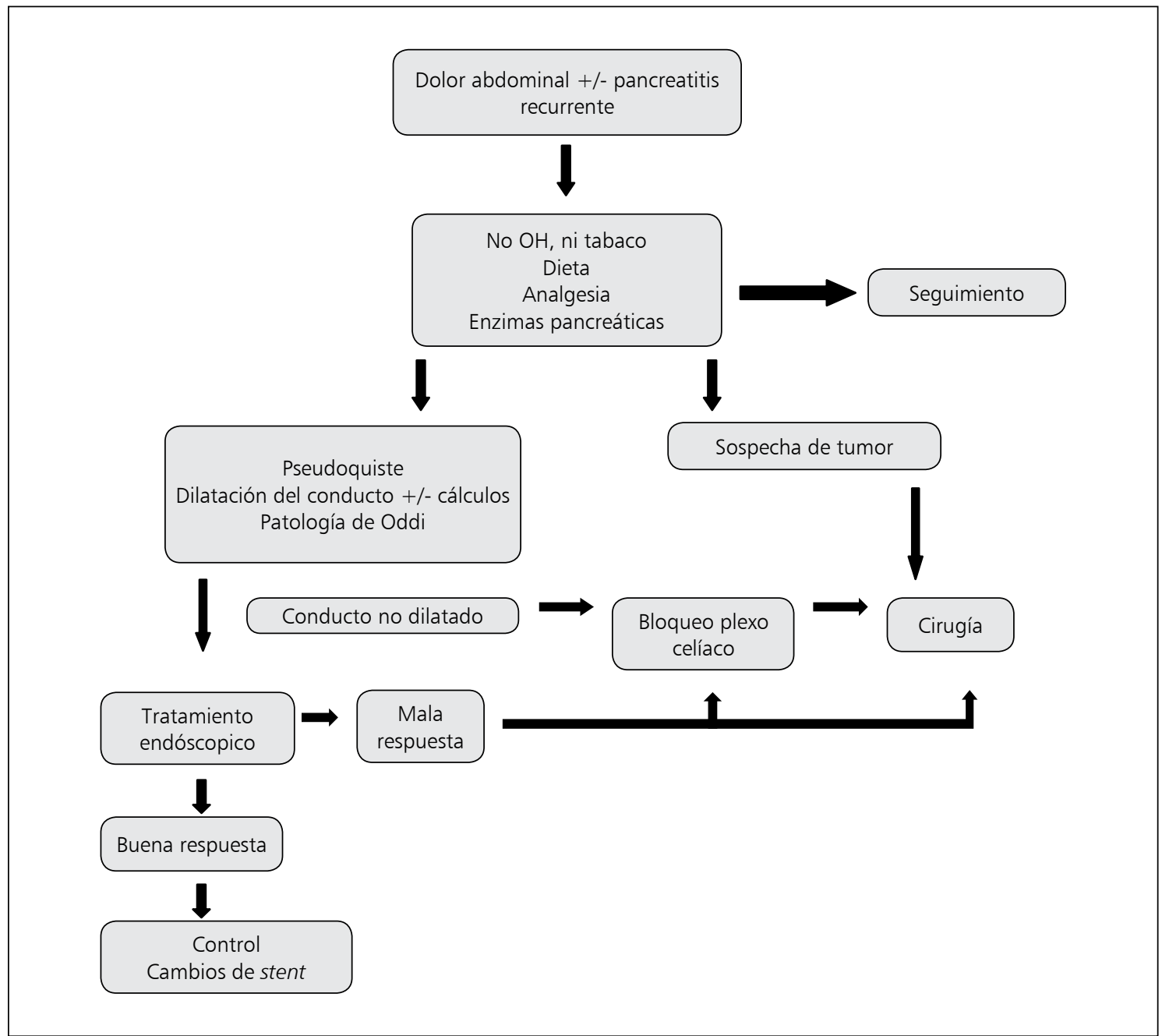

Figura 2. Algoritmo sugerido en el tratamiento en PC. El primer paso en caso de dolor abdominal, episodios agudos de pancreatitis es el respeto de dieta, abstinencia, tratamiento médico. Si fracasa, se obtienen imágenes actualizadas de páncreas. En caso de sospecha importante de tumor de páncreas, resección quirúrgica es el paso siguiente. Otras alteraciones morfológicas permiten el tratamiento endoscópico. En caso de ausencia de dilatación del conducto, bloqueo del plexo celíaco puede dar mejores resultados. Fracaso clínico de tratamiento endoscópico o de neurolísis, nuevamente la cirugía es el último recurso. 


\section{Referencias}

1. Domínguez-Muñoz JE. Pancreatitis Crónica. Gastroenterol Hepatol 2011; 34 Suppl 2: 78-81.

2. Etemad B, Whitcomb DC. Chronic pancreatitis: diagnosis, classification, and new genetic developments. Gastroenterology 2001; 120: 682-707.

3. Bockman DE, Boydston WR, Anderson MC. Origin of tubular complexes in human chronic pancreatitis. Am J Surg 1982; 144: 243-9.

4. DiMagno MJ, DiMagno EP. Chronic pancreatitis. Curr Opin Gastroenterol 2009; 25: 454-9.

5. Braganza JM, Lee SH, McCloy RF, McMahon MJ. Chronic pancreatitis. Lancet 2011; 377: 1184-97.

6. Oza VM, Kahaleh M. Endoscopic management of chronic pancreatitis. World J Gastrointest Endosc 2013; 5: 19-28.

7. Brock C, Nielsen LM, Lelic D, Drewes AM. Pathophysiology of chronic pancreatitis. World J Gastroenterol 2013; 19: 7231-40.

8. Pitchumoni CS. Pathogenesis and managenent of pain in chronic pancreatitis. World J Gastroenterol 2000; 6: 490-6.

9. Sarles H. Cros RC, Bidart JM. A multicenter inquiry into the etiology of pancreatic diseases. Digestion 1979; 19: 110-25.

10. Ammann RW, Heitz PU, Klöppel G. Course of alcoholic chronic pancreatitis: a prospective clinicomorphological long-term study. Gastroenterology 1996; 111: 224-31.

11. Dumonceau JM, Macias-Gómez C. Endoscopic management of complications of chronic pancreatitis. World J Gastroenterol 2013; 19: 7308-15.

12. Ammann RW, Muellhaupt B. The natural history of pain in alcoholic chronic pancreatitis. Gastroenterology 1999; 116: 1132-40.

13. Strobel O, Büchler MW, Werner J. Surgical therapy of chronic pancreatitis: indications, techniques and results. Int J Surg 2009; 7: 305-12.

14. Hildebrand P, Dudertadt S, Czymek R, Bader FG, Roblick UJ, Bruch HP, et al. Different surgical strategies for chronic pancreatitis significantly improve long-term outcome: a comparative single center study. Eur J Med Res 2010; 15: 351-6.

15. Issa Y, van Santvoort HC, van Goor H, Cahen DL, Bruno MJ, Boermeester MA. Surgical and Endoscopic Treatment of Pain in Chronic Pancreatitis: A Multidisciplinary Update. Dig Surg 2013; 30: 35-50.

16. Liao Z, Jin G, Cai D, Sun X, Hu B, Wang X, et al. Guidelines: diagnosis and therapy for chronic pancreatitis. J Interv Gastroenterol 2013; 3: 133-6.
17. Yoo BM, Lehman GA. Update on endoscopic treatment of chronic pancreatitis. Korean J Intern Med 2009; 24: 169-79.

18. Choi EK, Lehman GA. Update on endoscopic management of main pancreatic duct stones in chronic calcific pancreatitis. Korean J Intern Med 2012; 27: 20-9.

19. Tandan M, Nageshwar Reddy D. Endotherapy in chronic pancreatitis. World J Gastroenterol 2013; 19: 615664.

20. Goodman AJ, Gress FG. The endoscopic management of pain in chronic pancreatitis. Gastroenterol Res Pract 2012; 2012: 860-79.

21. Weber A, Schneider J, Neu B, Meining A, Born P, von Delius S, et al. Endoscopic stent therapy in patients with chronic pancreatitis: a 5-year follow-up study. World J Gastroenterol 2013; 19: 715-20.

22. Rossi R, de Aretxabala X, Watkins G, Navarrete C, Vargas L, Accatino L, et al. Pancreatitis crónica; Una experiencia nacional reciente de su manejo quirúrgico. Rev Med Chile 1997; 125: 911-6.

23. Gobelet J, Navarrete C, Sáenz R. Esfinterotomía pancreática: cuándo y cómo. Gastroenterol Hepatol 2006; 29: 584-91.

24. Yadav D, Whitcomb DC. The role of alcohol and smoking in pancreatitis. Nat Rev Gastroenterol Hepatol 2010; 7: 131-45.

25. DiMagno MJ, DiMagno EP. Chronic pancreatitis. Curr Opin Gastroenterol 2010; 26: 490-8.

26. Olesen SS, Juel J, Graversen C, Kolesnikov Y, Wilder-Smith OH, Drewes AM. Pharmacological pain management in chronic pancreatitis. World J Gastroenterol 2013; 19: 7292-301.

27. Bockman DE, Buchler M, Malfertheiner P, Beger HG. Analysis of nerves in chronic pancreatitis. Gastroenterology 1988; 94: 1459-69.

28. Attasaranya S, Abdel Aziz AM, Lehman GA. Endoscopic management of acute and chronic pancreatitis. Surg Clin North Am 2007; 87: 1379-402, viii.

29. Adler DG, Lichtenstein D, Baron TH, Davila R, Egan JV, Gan SL, et al. The role of endoscopy in patients with chronic pancreatitis. Gastrointest Endosc 2006; 63: 9337.

30. Dumonceau JM, Delhaye M, Tringali A, Domínguez-Muñoz JE, Poley JW, Arvanitaki M, et al. Endoscopic treatment of chronic pancreatitis: European Society of Gastrointestinal Endoscopy (ESGE) Clinical Guideline. Endoscopy 2012; 44: 784-800.

31. Clarke B, Slivka A, Tomizawa Y, Sanders M, Papachristou GI, Whitcomb DC, et al. Endoscopic therapy is effective for patients with chronic pancreatitis. Clin Gastroenterol Hepatol 2012; 10: 795-802. 
32. Kaufman M, Singh G, Das S, Concha-Parra R, Erber J, Micames C, et al. Efficacy of endoscopic ultrasound-guided celiac plexus block and celiac plexus neurolysis for managing abdominal pain associated with chronic pancreatitis and pancreatic cancer. J Clin Gastroenterol
2010; 44: 127-34.

33. Shen Y, Liu M, Chen M, Li Y, Lu Y, Zou X. Covered metal stent or multiple plastic stents for refractory pancreatic ductal strictures in chronic pancreatitis: A systematic review. Pancreatology 2014; 14: 87-90. 